\title{
Deep Learning Based Multi-user Interference Cancellation Technology
}

\section{Changyun Zhang}

Department of Electronics and Information Engineering, Shenzhen University, Shenzhen, China

\section{Email address:}

1441731605@qq.com

\section{To cite this article:}

Changyun Zhang. Deep Learning Based Multi-user Interference Cancellation Technology. Science Discovery. Vol. 7, No. 6, 2019 , pp. $379-384$. doi: $10.11648 /$ j.sd.20190706.11

Received: November 4, 2019; Accepted: December 3, 2019; Published: December 9, 2019

\begin{abstract}
In the paper, I proposed a neural network-based solution to multiple access interference under the Multi-antenna Input and Multi-antenna Output (MIMO) communication system. In a model of the uplink and downlink of the multiuser MIMO system. In cases of multiple access interference, each transmitter were designed with neural networks, after the transmitted signal passes through the channel, detecting received signals at receivers designed by neural network. The model could eliminate the interference between different users. The neural network-designed model adopted Rician fading channel (including Rayleigh fading channel) and simulated the Symbol Error Rate (SER) performance of multiple users under different signal-noise ratios. With respect to SER, the solution improved system performance compared with the current multiple access interference cancellation technology. Therefore, communication systems designed with neural networks face a promising future in multiple access interference cancellation.
\end{abstract}

Keywords: MIMO, Neural Network, Multi-user Interference Cancellation Technology, Symbol Error Rate

\section{基于深度学习的多用户干扰消除技术}

\section{张常运}

深圳大学信息工程学院, 深圳, 中国

\section{邮箱}

1441731605@qq.com

摘要: 本文提出了一种在MIMO(Multiple-Input Multiple-Output, 多天线输入多天线输出)通信系统中基于神经网络解决 多用户干扰问题的方案。在多用户MIMO系统上行链路与下行链路的模型中，当存在多个用户相互干扰时，使用神经 网络设计模型中的每个发射机, 它们发射的信号经过信道之后, 在神经网络设计的接收机处对接收信号进行检测, 可 用该模型消除多用户之间的干扰。在由神经网络设计的模型中，信道使用的是莱斯衰落新道（包含瑞利衰落信道）, 并仿真了在不同信噪比下多个用户的SER(Symbol Error Ratio, 误符号率)性能。在SER性能上, 用该方案与现有的多用 户干扰消除技术相比较, 可以看出前者提升了系统的性能。所以, 在未来的通信中用神经网络设计通信系统来消除多 用户干扰是一个很有潜力的方向。

关键词：MIMO，神经网络，多用户干扰消除技术，误符号率 


\section{1. 引言}

随着互联网蓬勃发展, 移动通信中的数据流量会大量 增加, 能源的消耗也会变得日益严重, 所以在未来的通信 中面临着降低成本、提高性能的挑战。随着用户数量的增 多, 解决它们之间的干扰成为了一个重要的研究方向, 如 Atsuto Kawagoe等人在多用户全双工系统中研究了用户间 干扰抑制方法 (波束形成方法和非线性方法) [1]; Weidong $\mathrm{Gao}$ 等人在迫零算法的基础上提出了一种协调的迫零波束 形成聚类方案 [2], 它结合了聚类算法和迫零算法[3]的优 点。Xianzhong Xie等人在下行链路多用户多小区的MIMO 系统[4]中, 利用基于干扰对齐的用户间干扰和小区间干扰 来设计收发器[5]。MIMO系统能充分利用空间资源, 通过 多个天线实现多发多收，在不增加频谱资源和天线发射功 率的情况下, 可以成倍的提高系统信道容量。但大规模多 用户MIMO系统的基站一般配备有数百个天线, 在相同的 时频资源上为数十个用户提供服务，所以Alexios Balatsoukas-Stimming 使用低精度数模转换器 (digital-to-analog converter, DAC) 降低系统的成本和功 耗, 提出了使用1位DAC的预编码算法 $\mathrm{C} 2 \mathrm{PO}$ (biConvex 1-bit PrecOding) [6]。

神经网络[7]是机器学习[8]中的一个重要方向, 它具 有自学习、自适应、自组织能力, 有较好的容错能力和处 理能力。将神经网络应用到多用户干扰消除中可显著降低 计算复杂度, 加快收玫速度。近年来越来越多的将神经网 络应用于各种通信任务 [9], 如调制识别、信号检测等问题, 大大减少了信号处理这种任务的时间。如Haoran Sun等人 研究了无线多用户干扰管理的问题 (特别是干扰信道的功 率控制问题），使用神经网络近似于干扰管理算法 WMMSE算法[10]。Tim O'Shea等人将通信系统视为端到 端的重建任务, 该任务旨在共同优化发送器和接收器组 件, 并将这种想法扩展到多个发射器和接收器的网络 [11]。 Tugba Erpek等人在文献[11]的基础上扩展了SISO系统, 使 用自动编码器神经网络架构模拟具有不同天线数量的 MIMO系统[12]。Michael Andri Wijaya等人在MIMO系统 中提出一种在发射机侧使用神经网络功率控制和在接收 机侧使用干扰消除的方案 [13], 很大的提高了系统的性能。 Neev Samuel[14]提出两种用于MIMO检测的深度学习网 络, 一是全连接层网络, 二是专门为MIMO检测设计的网 络。在每种场景下, 使用不同的信道模型和各种数字调制 方式, 他们设计的网络以低计算复杂度实现了接近最佳的 检测性能。

在本文中, 我在神经网络设计的系统中引入真实的信 道效应（瑞利信道与莱斯信道），扩展了文献[11]中的单 输入单输出 (SISO) 系统, 在MIMO系统中分别设计了上 行链路与下行链路的方案。把系统看为端到端的通信任 务, 在上行链路中有多个发射机和一个接收机, 在下行链 路中有一个发射机和多个接收机, 其中每个发射机和接收 机都用神经网络构建。多个用户共享同一信道并在训练期 间优化它们, 以便利用神经网络消除它们之间的干扰。最 后得出每个用户的误符号率性能，再用该通信系统模型的 性能与基于多用户检测技术 [15]的传统通信系统的性能进 行比较, 可以看出该方案显著提高了系统的性能。
本文的其他部分如下, 在第二部分介绍了信道模型以 及上下行链路的系统模型; 第三部分为基于神经网络的发 送接收机方案, 提供了有关基于神经网络的通信系统和我 所使用的深度学习算法的信息; 第四部分为数值仿真和性 能分析; 在第五部分提出了结论。

\section{2. 系统模型}

\section{1. 信道模型}

由于现实中的高楼林立, 在移动通信的传播环境中会 有很多的障碍物, 则移动台和基站之间的障碍物会产生的 反射、绕射和散射，接收信号通常由多径信号组成。所以 在该系统中使用的信道为瑞利信道与莱斯信道。当信道为 莱斯信道时, 信道响应为:

$$
h=\sqrt{\frac{q}{q+1}} h_{L O S}+\sqrt{\frac{1}{q+1}} h_{N L O S}
$$

其中 $q 、 h_{N L O S} 、 h_{L O S}$ 分别为莱斯因子、非直射路径的 分量与直射路径的分量。 $h_{N L O S}$ 服从均值为 0 , 方差为 1 的 复高斯过程。直射路径的分量 $h_{L O S}$ 为:

$$
h_{L O S}=e^{-j(m-1) \frac{2 \pi d}{\lambda} \sin \theta_{k}}
$$

其中 $m$ 代表基站处第 $m$ 根天线; $d$ 为天线之间的距 离; $\lambda$ 为电磁波波长, 并且 $\lambda=2 d ; \theta_{k}$ 为第 $k$ 个用户到 基站的到达角。

瑞利信道为莱斯信道的一种特殊形式, 它只有非直射 分量没有直射分量, 所以瑞利信道就是服从均值为 0 , 方 差为 1 的复高斯过程。

\section{2. 上行链路}

如图1(a)上行链路系统模型，共有 $K$ 个单天线用户， 所以该模型由 $K$ 个发射机和一个接收机组成。想要发送 的信息为 $M$ 个可能的符号 $s \in\{1,2,3, \cdots, M\}$ ，那么发送 符号 $s_{k}, k \in\{1,2, \cdots, K\}$ 为第 $k$ 个用户想要发送的符号。 $x_{k}, k \in\{1,2, \cdots, K\}$ 为 $s_{k}$ 经过发射机后的传输信号, 并对 他们进行平均功率约束。

信道响应为 $h_{k}, k \in\{1,2, \cdots, K\}, n$ 为均值为 0 , 方 差为 $\beta$ 的复高斯加性白噪声, 接收信号 $y^{u}$ 为:

$$
y^{u}=\sum_{i=1}^{K} h_{i}^{\mathrm{H}} x_{i}+n
$$

在接收机处对接收信号 $y^{u}$ 检测得到对 $s_{k}$ 的估计为 $\hat{s}_{k}, k \in\{1,2, \cdots, K\}$ 


\section{3. 下行链路}

如图1(b)下行链路系统模型, 共有 $K$ 个单天线用户, 所以该模型由一个发射机和 $K$ 个接收机组成。发送 $M$ 个可能的符号 $s \in\{1,2,3, \cdots, M\}, s_{k}$ 为第 $k$ 个用户想要 接收的符号，在发射机处同时发送 $K$ 个符号。这些符号 经过一个发射机后得到传输信号 $x$, 并对其进行平均功 率约束。 $h_{k}$ 为信道响应, $y_{k}^{l}, k \in\{1,2, \cdots, K\}$ 为接收信 号:

$$
y_{k}^{l}=h_{k}^{\mathrm{H}} x+n_{k}, k \in\{1,2, \cdots, K\}
$$

在接收机处对接收信号 $y_{k}^{l}$ 检测得到对 $s_{k}$ 的估计为 $\hat{s}_{k}$ 。

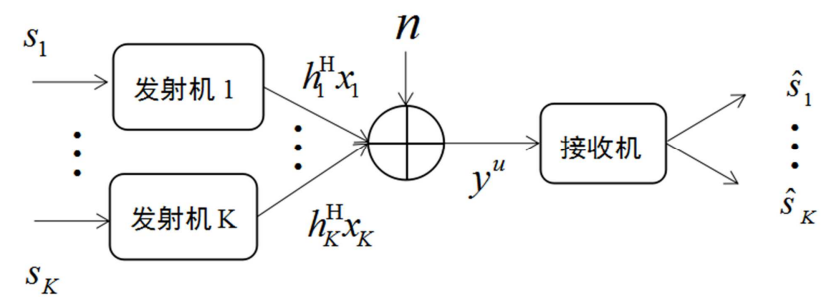

(a)

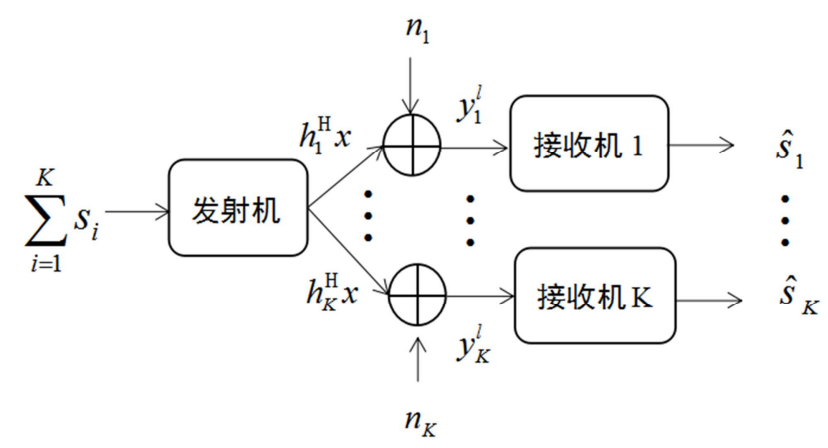

(b)

图1 系统模型。

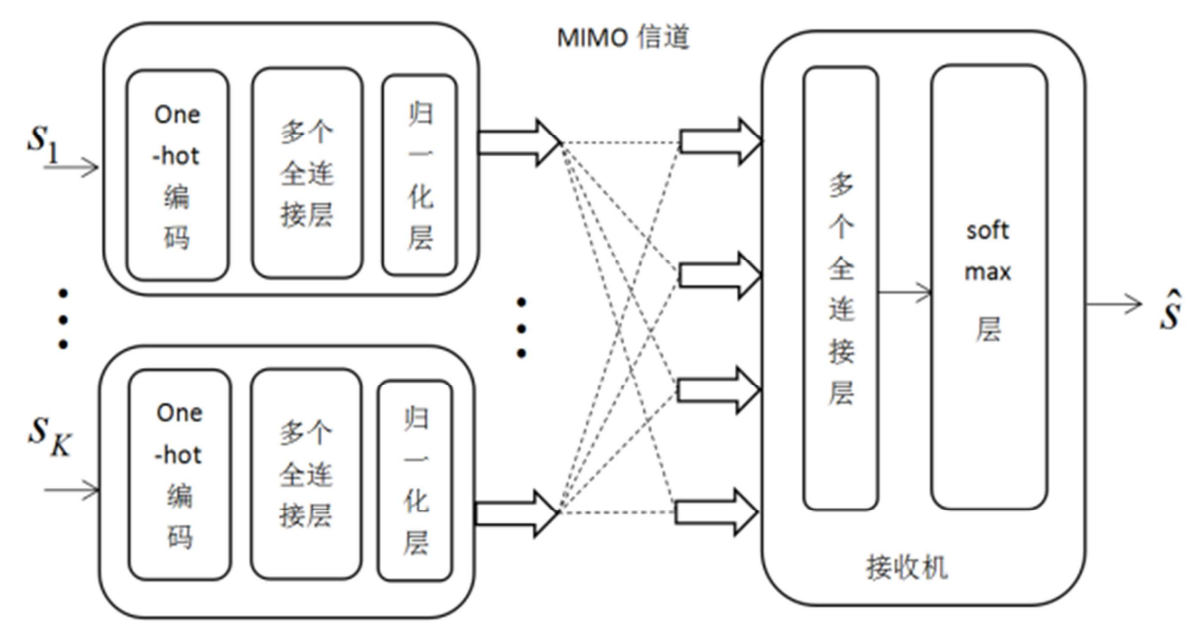

(a)

\section{3. 基于神经网络的发送接收机设计}

\section{1. 上行链路}

在MIMO系统中, 由于是多天线输入多天线输出, 所 以上行链路与下行链路的神经网络设计是不同的。如图 2(a)所示, 该模型为上行链路MIMO系统, 存在来自不同 发射机多个天线元件的干扰，这增加了系统的复杂性，所 以需要用神经网络设计更加复杂的发送机和接收机对信 号进行发送和检测。

在发射端处有 $K$ 个发射机, 在每个发射机处首先对 每一个发送符号进行One-hot编码, 因为要发送的可能的 符号有 $M$ 个, One-Hot向量的长度为 $M$, 想要发送的符 号 $S$ 对应于One-Hot向量中索引为 $S$ 的元素为 1 , 其他元素 全为 0 。之后是多层全连接神经网络, 其中每一层网络的 激活函数为Relu函数 (Rectified Linear Unit, 整流线性单 元）, Relu函数的表达式为:

$$
f(t)=\max (0, t)
$$

ReLu函数会使一部分神经元的输出为 0 , 这样就造成 了网络的稀疏性, 缓解了过拟合问题的发生。归一化层是 对传输信号进行功率约束。

在MIMO信道处引入了更加真实的信道效应（已在上 文中阐述), 令它与传输信号相乘, 再把所有的乘积相加, 还要加上一个复高斯噪声, 就得到了接收信号。

在接收机处有多根天线接收信号, 把接收到的信号进 行处理。也使用了多层神经网络, 除了最后一层神经网络 没有激活函数, 其他几层的激活函数都为Relu函数。由于 该任务是一个多分类的任务, 所以在最后一层接上了一个 softmax层:

$$
\operatorname{softmax}(\mathbf{z})_{i}=\frac{e^{z_{i}}}{\sum_{j} e^{z_{j}}}
$$

其中 $\mathbf{Z}$ 为最后一层神经网络的输出, softmax函数就是 对 $\mathbf{z}$ 指数化和归一化来得到最后的输出, 该输出向量中的 每个元素实际上就是希望输出的某个分类的概率。 


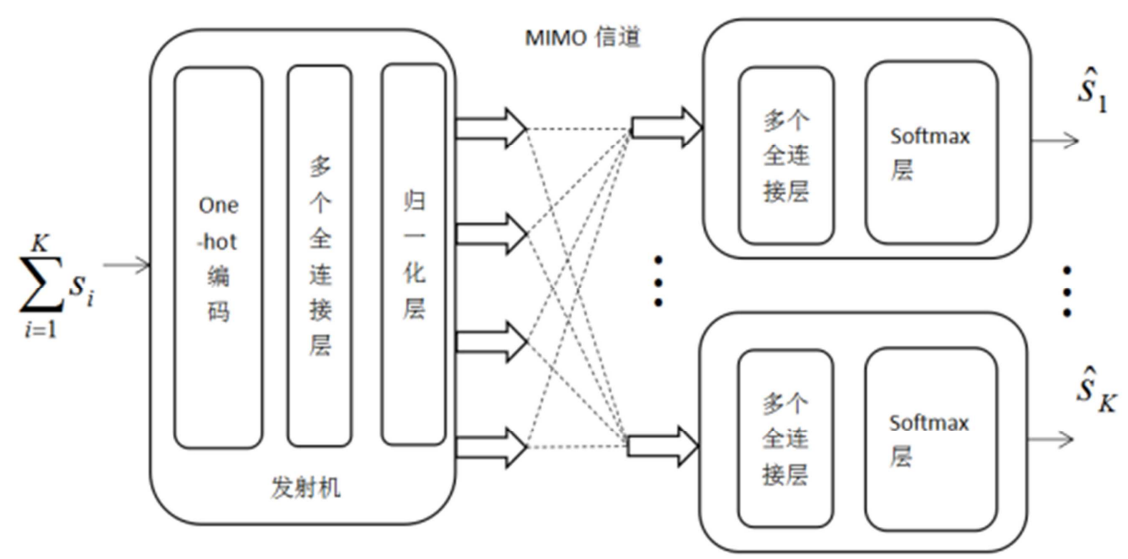

(b)

图2 神经网络--上下行链路MIMO系统。

\section{2. 下行链路}

如图2(b)所示, 该模型为下行链路MIMO系统。与 上行链路不同的是在发射端只有一个发射机, 先对每个 用户发送的符号进行One-Hot编码, 再令所有的One-Hot 向量相加, 把它作为神经网络的输入。经过多层全连接 神经网络, 其中每一层网络的激活函数为Relu函数。对 神经网络的输出归一化后, 把输出信号经过多根发射天 线发射出去。

在MIMO信道中引入真实地信道响应, 噪声为复高斯 噪声。在接收端有 $K$ 个接收机接收信号, 其中每个接收 机都由多层神经网络组成, 激活函数使用的是Relu函数, 并且也如同上行链路在最后一层神经网络上连接一个 softmax层。

\section{3. 优化}

在优化过程，由于将发射符号 $S$ 表示为长度为 $M$ 的 One-Hot矢量, 期望符号在One-Hot矢量中对应的元素值为 1。并且在神经网络设计的系统最后一层为softmax层, 输 出是一个 $M$ 维的矢量, 矢量中的每个元素为每个发送符 号可能的概率。则上述系统可以转化为多分类任务。在这 个分类任务中损失函数使用的是交叉熵损失函数

$$
\operatorname{Loss}(s, \hat{s})=\frac{-1}{M} \sum_{i=0}^{M-1}\left(s_{i} \log \left(\hat{s}_{i}\right)\right)
$$

其中 $\hat{s}$ 可以表示为: $\hat{s}=f(s, \theta)$, 其中 $\boldsymbol{\theta}$ 为神经网络 的参数, 包括权重和偏置, 为了进一步提高训练性能, 我 使用了截断的正态分布初始化权重。在反向传播中, 优化 算法使用的是梯度下降算法, 使损失函数最小化来优化网 络参数 $\theta$,

$$
\frac{\partial L}{\partial \theta}=\frac{\partial L(s, f(s, \theta))}{\partial \theta}
$$

参数更新 $\delta \theta=-\eta \frac{\partial L}{\partial \theta}$, 其中 $\eta$ 为学习率。

\section{4. 性能分析}

令基站处的天线数为 4 , 发射的符号数量 $M=4$ 。训 练数据为 10000 个, 测试数据为 400000 个, 其中每个数据 都是在 0 到 3 之间的随机数。并在不同的SNR (0dB-- $20 \mathrm{~dB})$ 下评估每个模型的SER性能。并将其与在相同条件下的基 于多用户检测SER性能进行比较, 在上行链路中使用的多 用户检测是MMSE (Minimum Mean Square Error, 最小均 方误差) 算法, 在下行链路中使用的是SIC (Successive Interference Cancellation，串行干扰消除）算法。

如表 1 与表 2 为神经网络设计的上行链路与下行链路 中每层网络的输出维度。

表1 每一层的输出维度（上行链路MIMO系统）。

\begin{tabular}{ll}
\hline 每一层 & 输出维度 \\
\hline 输入层 & $\mathrm{M}$ \\
全连接层+Relu & 8 \\
全连接层 & 10 \\
归一化层 & 10 \\
噪声层 & 40 \\
全连接层+Relu & 20 \\
全连接层+Relu & 8 \\
全连接层+softmax & $\mathrm{M}$ \\
\hline
\end{tabular}

表2 每一层的输出维度（下行链路MIMO系统）。

\begin{tabular}{ll}
\hline 每一层 & 输出维度 \\
\hline 输入层 & $\mathrm{M}$ \\
全连接层+Relu & 20 \\
全连接层 & 40 \\
归一化层 & 40 \\
噪声层 & 10 \\
全连接层+Relu & 20 \\
全连接层+Relu & 8 \\
全连接层+softmax & $\mathrm{M}$ \\
\hline
\end{tabular}

当系统的用户数为 2 时, 如图 3 与图 4 分别为上行链路 与下行链路MIMO系统的SER性能, 其中的SER为在相同 的信噪比下对两个用户的误符号率求均值而得到的结果。 并且使用了瑞利信道与莱斯信道来对 SER性能进行评估。 
从上行链路与下行链路SER性能图中可以看出莱斯信道 MIMO系统比瑞利信道MIMO系统的SER性能要好, 但是 随着信噪比的增大, 它们的相差会变得越来越小。

在上行链路SER图中低信噪比时, 神经网络设计的系 统与基于多用户检测的系统SER性能相似, 但是随着信噪 比的增大二者的差距也会变大。在下行链路SER图中, 用 神经网络设计系统的SER性能明显更好。综上所述, 神经 网络设计的系统可以很好地消除了其他用户的干扰。

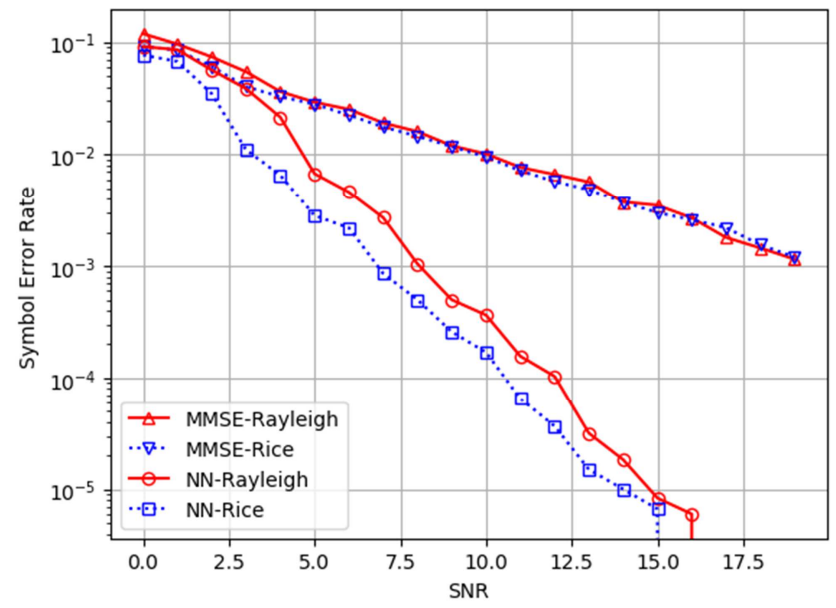

图3 上行链路的SER性能。

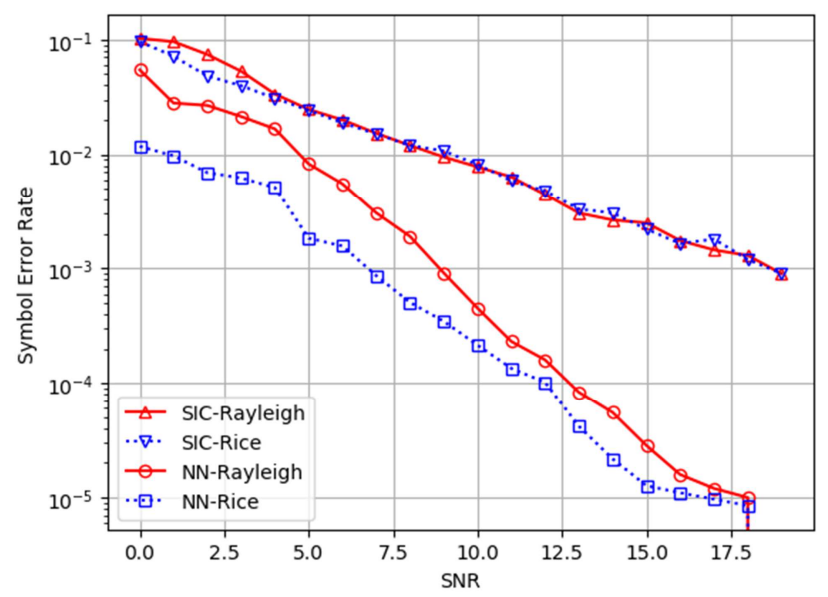

图4 下行链路的SER性能。

当用户数为 3 时, 如图 5 与图 6 分别为上行链路与下行 链路MIMO系统的SER性能。

其中的SER为在相同的信噪比下对三个用户的误符 号率求均值而得到的结果。莱斯信道MIMO系统的性能仍 然优于瑞利信道MIMO系统的SER性能。但是随着信噪比 的增大, 它们的SER性能会变得相近。

由神经网络设计的系统在性能上仍然优于基于多用 户检测的误符号率性能。但是相比较于两个用户时SER高 了一点, 由此可见随着用户数量的增多, 误符号率性能会 随之变差。

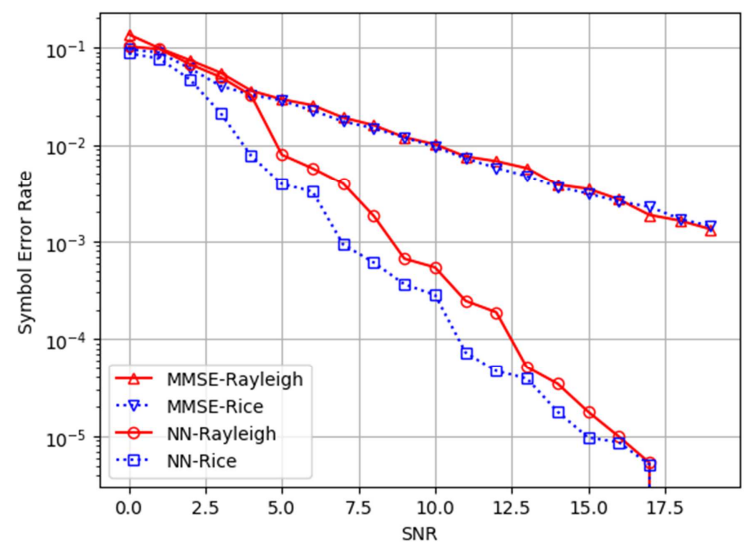

图5 上行链路的SER性能。

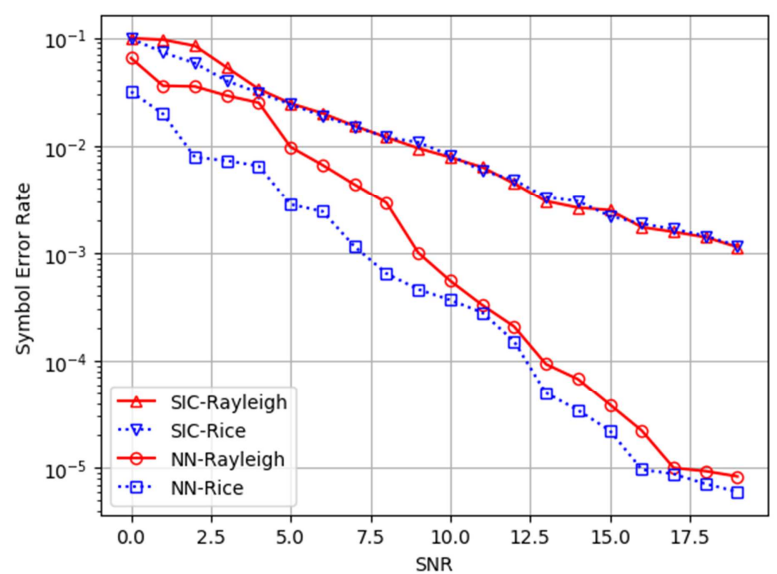

图6 下行链路的SER性能。

\section{5. 结论}

在本文中评估了在存在多用户干扰的情况下基于神 经网络MIMO系统的误符号率性能。我在衰落信道（瑞利 与莱斯信道) 上使用全连接神经网络构建了 $\mathrm{MIMO}$ 系统中 上行链路与下行链路的模型, 通过数据优化网络模型来最 小化SER, 并将其与基于多用户检测的SER进行比较, 可 以看出该模型提高了系统的性能。所以使用神经网络设计 通信系统是未来通信的一个重要方向。

但是我提出的这种架构对于天线数量的变化不够灵 活 (即, 基站处天线数量的任何变化都需要设计新的网 络）。下一步的工作就是需要考虑在不同天线数量的情况 下设计网络架构。尽管如此, 我认为这种方法仍然是一个 有潜力的方向, 并且有可能影响到未来的通信系统。

\section{参考文献}

[1] A. Kawagoe, N. Honma and N. Takemura, "Imp-act of user terminal antenna spacing on inter-terminal interference cancellation in multi-user full duplex MIMO transmission," 2017 IEEE 28th Annual International Symposium on Personal, Indoor, and Mobile Radio Communications (PIMRC), Montreal, QC, 2017, pp. 1-4. 
[2] W. Gao, S. He and G. Chuai, "Clusteringfor coordinated zero-forcing beamformingin multi-user interference networks," 2016 IEEE International Conference on Network Infrastructure and Digital Content (IC-NIDC), Beijing, 2016, pp. 322-326.

[3] 王晈,强永全,李道本. 迭代迫零多用户检测算法研究与应 用 $[\mathrm{J}]$. 无线电工程(11):11-13,19.

[4] P. P. Vaidyanathan, "On the degree of MIMO systems," 2007 IEEE International Symposium on Circuits and Systems, New Orleans, LA, 2007, pp. 661-664.

[5] X. Xie, H. Yang and A. V. Vasilakos, "Robust Transceiver Design Based on Interference Alignment for Multi-User Multi-Cell MIMO Networks With Channel Uncertainty," in IEEE Access, vol. 5, pp. 5121-5134, 2017.

[6] A. Balatsoukas-Stimming, O. Castañeda, S. Jacobsson, G. Durisi and C. Studer, "Neural-Network Optimized 1-bit Precoding for Massive MU-MIMO," 2019 IEEE 20th International Workshop on Signal Processing Advances in Wireless Communications (SPAWC), Cannes, France, 2019, pp. 1-5.

[7] I. Goodfellow, Y. Bengio, and A. Courville, Deep learning. MIT press, 2016.

[8] 王珏, 石纯一. 机器学习研究[J]. 广西师范大学学报(自然 科学版), 2003(02):4-18.

[9] C. Zhang, P. Patras and H. Haddadi, "Deep Learning in Mobile and Wireless Networking: A Survey," in IEEE
Communications Surveys \& Tutorials, vol. 21, no. 3, pp. 2224-2287, thirdquarter 2019.

[10] H. Sun, X. Chen, Q. Shi, M. Hong, X. Fu and N. D. Sidiropoulos, "Learning to Optimize: Training Deep Neural Networks for Interference Management," in IEEE Transactions on Signal Processing, vol. 66, no. 20, pp. 5438-5453, 15 Oct.15, 2018.

[11] T. O'Shea and J. Hoydis, "An Introduction to Deep Learning for the Physical Layer," in IEEE Transactions on Cognitive Communications and Networking, vol. 3, no. 4, pp. 563-575, Dec. 2017.

[12] T. Erpek, T. J. O'Shea and T. C. Clancy, "Learning a Physical Layer Scheme for the MIMO Interfere-nce Channel," 2018 IEEE International Conference on Communications (ICC), Kansas City, MO, 2018, pp. 1-5.

[13] M. A. Wijaya, K. Fukawa and H. Suzuki, "Intercell-Interference Cancellation and Neural Network Transmit Power Optimization for MIMO Channels," 2015 IEEE 82nd Vehicular Technology Conference (VTC2015-Fall), Boston, MA, 2015, pp. 1-5.

[14] N. Samuel, T. Diskin and A. Wiesel, "Learning to Detect," in IEEE Transactions on Signal Processing, vol. 67, no. 10, pp. 2554-2564, 15 May15, 2019.

[15] J. Yang and J. Song, "Multi-user detection under wireless channel," 2009 IEEE International Conference on Communications Technology and Applications, Beijing, 2009, pp. 581-585. 